\title{
GRADIENT ESTIMATES FOR THE HEAT EQUATION UNDER THE RICCI-HARMONIC MAP FLOW
}

\author{
MIHAI BĂILEŞTEANU
}

\begin{abstract}
The paper establishes a series of gradient estimates for positive solutions to the heat equation on a manifold $M$ evolving under the Ricci flow, coupled with the harmonic map flow between $M$ and a second manifold $N$. We prove Li-Yau type Harnack inequalities and we consider the cases when $M$ is a complete manifold without boundary and when $M$ is compact, without boundary.
\end{abstract}

\section{INTRODUCTION}

The present article focuses on the behaviour of positive solutions to the heat equation on a Riemannian manifold $M$, whose metric evolves under the Ricci flow coupled with the harmonic map flow of a map $\phi$ from $M$ to another manifold $N$. Our goal is to establish a series of Li-Yau type gradient estimates, which naturally lead to Harnack inequalities. The results are both of local and global nature, as we are studying the case when $M$ is complete without boundary and the case when $M$ is compact without boundary.

Given two Rimeannian manifolds $(M, g)$ and $(N, \gamma)$ and a map $\phi: M \rightarrow N$, the Ricciharmonic map flow is the coupled system of the Ricci flow with the harmonic map flow of $\phi$ :

$$
\left\{\begin{array}{l}
\frac{\partial}{\partial t} g(x, t)=-2 \operatorname{Ric}(x, t)+2 \alpha(t) \nabla \phi(x, t) \otimes \nabla \phi(x, t) \\
\frac{\partial}{\partial t} \phi(x, t)=\tau_{g} \phi(x, t)
\end{array}\right.
$$

where $\alpha$ is a positive coupling time-dependent function. $\tau_{g} \phi$ denotes the tension field of the map $\phi$ with respect to the metric $g(t)$. We will refer to it as the $(R H)_{\alpha}$ flow, for short and we call $(g(x, t), \phi(x, t))$ with $t \in[0, T]$ a solution to this flow. It is interesting that it may be less singular than both the Ricci flow (to which it reduces when $\alpha(t)=0$ ) and the harmonic map flow. We assume that the curvature of $M$ remains bounded for all $t \in[0, T]$. We also consider a function $u: M \times[0, T] \rightarrow(0, \infty)$ which solves the heat equation

$$
\left(\triangle-\frac{\partial}{\partial t}\right) u(x, t)=0, x \in M, t \in[0, T] .
$$

One can give a simple physical interpretation of problem (1.1) combined with (1.2): the manifold $M$ with initial metric $g(x, 0)$ can be thought as an object having the temperature distribution $u(x, 0)$. Then, simultaneously, $M$ starts evolving under the $(R H)_{\alpha}$ flow, while heat is spreading onto it. The solution $u(x, t)$ represents the temperature at time $t$ and point $x$.

Department of Mathematics, University of Rochester, 801 Hylan Bld, Rochester, NY 14627, USA mbailest@z.rochester.edu 
The $(R H)_{\alpha}$ flow was introduced in [1, where the author proved short time existence and studied energy and entropy functionals, existence of singularities, a local non-collapsing property etc. Moreover, a version of this flow appeared earlier in the work of List [2], where the case of $\phi$ being a scalar function and $\alpha=2$ was analyzed, and where it was shown to be equivalent to the gradient flow of an entropy functional, whose stationary points are solutions to the static Einstein vacuum equations.

The $(R H)_{\alpha}$ flow also arises when one studies Ricci flow on warped product spaces. Given a warped product metric $g_{M}=g_{N}+e^{2 \phi} g_{F}$ on a manifold $M=N \times F$ (where $\phi \in C^{\infty}(N)$ ), the Ricci flow equation on $M \frac{\partial g_{M}}{\partial t}=-2 \operatorname{Ric}_{M}$ leads to the following equations on each component:

$$
\left\{\begin{array}{l}
\frac{\partial g_{N}}{\partial t}=-2 \operatorname{Ric}_{N}+2 m d \phi \otimes d \phi \\
\frac{\partial \phi}{\partial t}=\triangle \phi-\mu e^{-2 \phi}
\end{array}\right.
$$

if the fibers $F$ are $m$-dimensional and $\mu$-Einstein. This is a particular version of the $(R H)_{\alpha}$, where the target manifold is one dimensional, and has been studied by M. B. Williams in [3] and by H. Tran in [4] (when $\mu=0)$.

The scalar curvature of a manifold evolving under the $(\mathrm{RH})_{\alpha}$ flow satisfies the heat equation with a potential (depending on the Ricci curvature of $M$, the map $\phi$ and the Riemann curvature tensor of $N$ ), so in order to understand the behavior of the metric under the $(R H)_{\alpha}$ flow one needs to study the heat equation.

The study of the heat equation under the Ricci flow started with R. Hamilton's paper [5], and later it was pursued in [6, 7, 8, 9, 5]. Most recently, gradient estimates for the heat equation under the Ricci flow were analyzed in [10, [11] and [12].

The main two results of this paper are the following two theorems.

Theorem 1.1. Let $(g(x, t), \phi(x, t))_{t \in[0, T]}$ is a solution to the $(R H)_{\alpha}$ flow (1.1), where the manifold $M^{n}$ is compact and $\alpha(t)$ is a non-increasing function in time, bounded from below by $\bar{\alpha}$. Assume that $0 \leq \operatorname{Ric}(x, t) \leq k g(x, t)$ for some $k>0$ and all $(x, t) \in M \times[0, T]$. Moreover suppose that $0 \leq \nabla_{i} \phi \nabla_{j} \phi \leq \frac{C}{t} g_{i j}$, for all $i, j \in\{1,2, \ldots, n\}$ ( $C$ being a constant depending on $n$ and $\bar{\alpha})$. Let $u$ be a smooth positive function $u: M \times[0, T] \rightarrow \mathbb{R}$ satisfying the heat equation $u_{t}=\triangle u$. The estimate

$$
\frac{|\nabla u|^{2}}{u^{2}}-\frac{u_{t}}{u} \leq k n+\frac{C_{n}}{2 t}
$$

holds for all $(x, t) \in M \times(0, T]$, where $C_{n}=n / 2+4 n C \alpha(0)$.

This estimate resembles the Li-Yau inequality from [13], where the authors found that the solution to the heat equation (1.2) on a static manifold (with non-negative Ricci curvature) satisfies:

$$
\frac{|\nabla u|^{2}}{u^{2}}-\frac{u_{t}}{u} \leq \frac{n}{2 t}
$$

and the expression becomes equality when $u(x, t)$ is the heat kernel on the Euclidean space.

The second result is local in nature and is achieved inside the ball $B_{\rho, T}=\{(x, t) \in$ $\left.M \times[0, T] \mid \operatorname{dist}\left(x, x_{0}, t\right)<\rho\right\}$, while requiring $M$ only to be complete:

Theorem 1.2. Let $(g(x, t), \phi(x, t))_{t \in[0, T]}$ be a solution to the $(R H)_{\alpha}$ flow (1.1), where $M^{n}$ is complete and $\alpha(t)$ is a non-increasing function in time, bounded from below by $\bar{\alpha}$. Suppose $-k_{1} g(x, t) \leq \operatorname{Ric}(x, t) \leq k_{2} g(x, t)$ for some $k_{1}, k_{2}>0$ and all $(x, t) \in B_{\rho, T}$ and that $0 \leq$ 
$\nabla_{i} \phi \nabla_{j} \phi \leq \frac{C}{t} g_{i j}$, for all $i, j \in\{1,2, \ldots, n\}$ (C being a constant depending on $n$ and $\left.\bar{\alpha}\right)$. Consider a smooth positive function $u: M \times[0, T] \rightarrow \mathbb{R}$ solving the heat equation $\triangle u=u_{t}$. There exists a constant $C^{\prime}$ that depends on the dimension of $M, \alpha(0)$, and $C$ and satisfies the estimate

$$
\frac{|\nabla u|^{2}}{u^{2}}-\beta \frac{u_{t}}{u} \leq C^{\prime} \beta^{2} \cdot\left(\frac{\beta^{2}}{\rho(\beta-1)}+\frac{1}{t}+\max \left\{k_{1}, k_{2}\right\}\right)+\frac{n \beta k_{1}}{4(\beta-1)}
$$

for all $\beta>1$ and all $(x, t) \in B_{\frac{\rho}{2}, T}$ with $t \neq 0$.

The proof of the theorems are given in section 2.2 and 2.3, respectively. We conclude the paper with section 3, where we present the Harnack inequalities resulting from integrating the above gradient estimates over space-time paths.

\section{Gradient estimates}

2.1. Preliminaries. First, let us note that $M$ is a connected, oriented, smooth, $n$ dimensional Riemannian manifold, without boundary, with metric $g$. Similarly $N$ is also a smooth manifold, without boundary, of dimension $m$. We assume that $N$ is isometrically embedded into the Euclidean space $\mathbb{R}^{d}$ (which follows by Nash's embedding theorem) for large enough $d$, so one may write $\phi=\left(\phi^{\mu}\right)_{1 \leq \mu \leq d}$.

We will assume that $(\phi(x, t), g(x, t))$ is a solution to the $(R H)_{\alpha}$ flow (1.1), for $x \in M$ and $t \in[0, T]$, where $T<T_{\epsilon}$, where $T_{\epsilon}$ is the time when there is possibly a blowup in the metric.

The tensor $\nabla \phi(x, t) \otimes \nabla \phi(x, t)$ has the following expression in local coordinates: $(\nabla \phi \otimes$ $\nabla \phi)_{i j}=\nabla_{i} \phi^{\mu} \nabla_{j} \phi^{\mu}$ and the energy density of the map $\phi$ is given by $|\nabla \phi|^{2}=g^{i j} \nabla_{i} \phi^{\mu} \nabla_{j} \phi^{\mu}$, where we use the convention that repeated Latin indices are summed over from 1 to $n$, while the Greek are summed from 1 to $d$. All the norms are taken with respect to the metric $g$ at time $t$.

As we mentioned in the introduction, we will assume the most general condition for the coupling function $\alpha(t)$, as it appears in [1]: it is a non-increasing function in time, bounded from below by $\bar{\alpha}>0$, at any time.

Note that in [1] (Proposition 5.5), the author proves that if $N$ has non-positive sectional curvature, then

$$
|\nabla \phi(x, t)|^{2} \leq \frac{n}{2 \bar{\alpha} t}, \forall(x, t) \in M \times(0, T]
$$

where $n$ is the dimension of $M$ and $[0, T]$ is the interval in which the flow is defined $\left(T<T_{e}\right.$, where $T_{e}$ is the moment when there is possibly a blowup).

Both of our theorems hold under a slightly stronger assumption: $0 \leq \nabla_{i} \phi \nabla_{j} \phi \leq \frac{C}{t} g_{i j}$, for all $i, j \in\{1,2, \ldots, n\}$ ( $C$ being a constant depending on $n$ and $\bar{\alpha}$ ), which immediately implies the above inequality. As a future endeavor, one may want to prove the same theorems without this assumption.

Following the notation in [1], it will be easier to introduce these quantities:

$$
\begin{aligned}
\mathcal{S} & :=\operatorname{Ric}-\alpha \nabla \phi \otimes \nabla \phi \\
S_{i j} & :=R_{i j}-\alpha \nabla_{i} \phi \nabla_{j} \phi \\
S & :=R-\alpha|\nabla \phi|^{2}
\end{aligned}
$$

Both proofs are based on the following lemma: 
Lemma 2.1. Suppose $(g(x, t), \phi(x, t))_{t \in[0, T]}$ is a solution to the $(R H)_{\alpha}$ flow (1.1). Assume that $-k_{1} g(x, t) \leq \operatorname{Ric}(x, t) \leq k_{2} g(x, t)$ for some $k_{1}, k_{2}>0$ and that $0 \leq \nabla_{i} \phi \nabla_{j} \phi \leq \frac{C}{t} g_{i j}$, for all $i, j \in\{1,2, \ldots, n\}$ (C being a constant depending on $n$ and $\bar{\alpha})$. Suppose $u: M \times[0, T] \rightarrow \mathbb{R}$ is a smooth positive function satisfying the heat equation $u_{t}=\Delta u$. Given $\beta \geq 1$, define $f=\log u$ and $F=t\left(|\nabla f|^{2}-\beta f_{t}\right)$. The estimate

$$
\begin{aligned}
\left(\triangle-\frac{\partial}{\partial t}\right) F \geq & -2 \nabla f \nabla F+\frac{2 a \beta t}{n}\left(|\nabla f|^{2}-f_{t}\right)^{2} \\
& -\left(|\nabla f|^{2}-\beta f_{t}\right)-2 k_{1} \beta t|\nabla f|^{2}-\frac{\beta t n}{2 b} \max \left\{k_{1}^{2}, k_{2}^{2}\right\} \\
& -\frac{\beta \alpha^{2}(0) n}{2 b} \frac{C^{2}}{t}-2(\beta-1) \alpha(0) C|\nabla f|^{2}
\end{aligned}
$$

holds for any $a, b>0$ such that $a+2 b=\frac{1}{\beta}$.

Proof. Since $u_{t}=\Delta u$ and $f=\log u$, then $f_{t}=\Delta f+|\nabla f|^{2}$. Also, note that

$$
\begin{aligned}
\left(|\nabla f|^{2}\right)_{t} & =2 S_{i j} \nabla_{i} f \nabla_{j} f+2 \nabla f \cdot \nabla\left(f_{t}\right) \\
(\triangle f)_{t} & =2 S_{i j} \nabla_{i} \nabla_{j} f+\triangle\left(f_{t}\right) \\
\triangle \nabla_{i} f & =\nabla_{i} \triangle f+R_{i j} \nabla_{j} f \\
\triangle|\nabla f|^{2} & =2|\nabla \nabla f|^{2}+2 R_{i j} \nabla_{i} f \nabla_{j} f+2 \nabla_{i} f \nabla_{i}(\triangle f)
\end{aligned}
$$

The first two follow from the flow equation, while the last two are Bochner identities.

Let's denote $\nabla_{i} f:=f_{i}$ and $\nabla_{i} \nabla_{j} f:=f_{i j}$.

Using these, one gets that

$$
\triangle F-F_{t}=-2 \nabla f \cdot \nabla F-\left(|\nabla f|^{2}-\beta f_{t}\right)+2 t\left(f_{i j}^{2}+\beta S_{i j} f_{i j}\right)+2 t R_{i j} f_{i} f_{j}+2 t(\beta-1) S_{i j} f_{i} f_{j}
$$

The last two terms can be bounded as follows:

$$
\begin{aligned}
2 t R_{i j} f_{i} f_{j}+2 t(\beta-1) S_{i j} f_{i} f_{j} & =2 t \beta R_{i j} f_{i} f_{j}-2 t(\beta-1) \alpha(t) \nabla_{i} \phi \nabla_{j} \phi f_{i} f_{j} \\
& \geq-2 t \beta k_{1}|\nabla f|^{2}-2(\beta-1) \alpha(0) C|\nabla f|^{2}
\end{aligned}
$$

Suppose at time $t$, at the point $x$ where we are estimating the quantity, we choose normal coordinates associated to the Levi-Civita connection. Then at this point the metric $g$ is diagonal (and hence so is Ric), in fact it is the identity, and has zero directional derivatives. However, that doesn't mean that $\nabla \phi \otimes \nabla \phi$ can also be diagonalized. But this point $g_{i j}=g^{i j}$ and hence $\alpha(t) \nabla_{i} \phi \nabla_{j} \phi f_{i} f_{j} \leq \alpha(0) \frac{C}{t} g^{i j} f_{i} f_{j}=\alpha(0) C|\nabla f|^{2}$.

The term $f_{i j}^{2}+\beta S_{i j} f_{i j}$ needs to be dealt separately:

$$
\begin{aligned}
f_{i j}^{2}+\beta S_{i j} f_{i j} & =f_{i j}^{2}+\beta R_{i j} f_{i j}-\beta \alpha \nabla_{i} \phi \nabla_{j} \phi f_{i j} \\
& =(a+2 b) \beta f_{i j}^{2}+\beta R_{i j} f_{i j}-\beta \alpha \nabla_{i} \phi \nabla_{j} \phi f_{i j} \\
& =a \beta f_{i j}^{2}+\beta\left(\sqrt{b} f_{i j}+\frac{R_{i j}}{2 \sqrt{b}}\right)-\frac{\beta}{4 b} R_{i j}^{2}+\beta\left(\sqrt{b} f_{i j}+\frac{\alpha \nabla_{i} \phi \nabla_{j} \phi}{2 \sqrt{b}}\right)-\frac{\beta}{4 b}\left(\alpha \nabla_{i} \phi \nabla_{j} \phi\right)^{2} \\
& \geq a \beta f_{i j}^{2}-\frac{\beta}{4 b} R_{i j}^{2}-\frac{\beta}{4 b}\left(\alpha \nabla_{i} \phi \nabla_{j} \phi\right)^{2} \\
& \geq a \beta f_{i j}^{2}-\frac{\beta n k^{2}}{4 b}-\frac{\beta \alpha^{2}(0) n C^{2}}{4 b t^{2}}
\end{aligned}
$$

where $k=\max \left\{k_{1}, k_{2}\right\}$. 
Note that because $\nabla_{i} \phi \nabla_{j} \phi \leq \frac{C}{t} g_{i j}$, then $\left(\nabla_{i} \phi \nabla_{j} \phi\right)^{2} \leq \frac{C}{t} g_{i j} g^{i j}=\frac{n C^{2}}{t^{2}}$ since we are using normal coordinates $\left(g_{i j}=g^{i j}\right)$.

Finally, let's observe that $\sum f_{i j}^{2} \geq \frac{(\triangle f)^{2}}{n}=\frac{\left(f_{t}-|\nabla f|^{2}\right)^{2}}{n}$.

Putting all together, we obtain:

$$
\begin{aligned}
\triangle F-F_{t} & \geq-2 \nabla f \cdot \nabla F-\left(|\nabla f|^{2}-\beta f_{t}\right)+\frac{2 a \beta t}{n}\left(|\nabla f|^{2}-f_{t}\right)^{2}-2 k_{1} \beta t|\nabla f|^{2} \\
& -\frac{\beta t n}{2 b} \max \left\{k_{1}^{2}, k_{2}^{2}\right\}-\frac{\beta \alpha^{2}(0) n}{2 b} \frac{C^{2}}{t}-2(\beta-1) \alpha(0) C|\nabla f|^{2}
\end{aligned}
$$

The proofs of Theorem 1.2 will involve a cut-off function on $B_{\rho, T}$. The construction of this function will rely on the following well-known lemma. It was previously used in the proofs of Theorems 2.3 and 3.1 in [6]; see also [14, Chapter IV] and [15].

Lemma 2.2. Given $\tau \in(0, T]$, there exists a smooth function $\bar{\Psi}:[0, \infty) \times[0, T] \rightarrow \mathbb{R}$ satisfying the following requirements:

(1) The support of $\bar{\Psi}(r, t)$ is a subset of $[0, \rho] \times[0, T]$, and $0 \leq \bar{\Psi}(r, t) \leq 1$ in $[0, \rho] \times[0, T]$.

(2) The equalities $\bar{\Psi}(r, t)=1$ and $\frac{\partial \bar{\Psi}}{\partial r}(r, t)=0$ hold in $\left[0, \frac{\rho}{2}\right] \times[\tau, T]$ and $\left[0, \frac{\rho}{2}\right] \times[0, T]$, respectively.

(3) The estimate $\left|\frac{\partial \bar{\Psi}}{\partial t}\right| \leq \frac{\bar{C} \bar{\Psi} \frac{1}{2}}{\tau}$ is satisfied on $[0, \infty) \times[0, T]$ for some $\bar{C}>0$, and $\bar{\Psi}(r, 0)=$ 0 for all $r \in[0, \infty)$.

(4) The inequalities $-\frac{C_{a} \bar{\Psi}^{a}}{\rho} \leq \frac{\partial \bar{\Psi}}{\partial r} \leq 0$ and $\left|\frac{\partial^{2} \bar{\Psi}}{\partial r^{2}}\right| \leq \frac{C_{a} \bar{\Psi}^{a}}{\rho^{2}}$ hold on $[0, \infty) \times[0, T]$ for every $a \in(0,1)$ with some constant $C_{a}$ dependent on $a$.

\subsection{Proof of Theorem 1.1.}

Proof. Let $f=\log u$. Denote $F=t\left(|\nabla f|^{2}-f_{t}\right)$. Fix $\tau \in(0, T]$ and choose a point $\left(x_{0}, t_{0}\right) \in M \times[0, \tau]$ where $F$ attains its maximum on $M \times[0, \tau]$. We will show that

$$
F\left(x_{0}, t_{0}\right) \leq t_{0} k n+\frac{C_{n}}{2} .
$$

If $t_{0}=0$, then $F\left(x, t_{0}\right)$ is equal to 0 for every $x \in M$ and the estimate is trivially true. Assume then that $t_{0}>0$ without loss of generality. We apply Lemma 2.1 with $k_{1}=0$, $k_{2}=k, \beta=1$ and $a+2 b=1$ and we obtain:

$$
\left(\Delta-\frac{\partial}{\partial t}\right) F \geq-2 \nabla f \nabla F+\frac{2 a}{n} \frac{F^{2}}{t_{0}}-\frac{F}{t_{0}}-\frac{t_{0} n}{(1-a)} k^{2}-\frac{\alpha^{2}(0) n}{1-a} \frac{C^{2}}{t_{0}}
$$

for all $a \in(0,1)$ at the point $\left(x_{0}, t_{0}\right)$.

$F$ attains its maximum at $\left(x_{0}, t_{0}\right)$, which implies that $\triangle F\left(x_{0}, t_{0}\right) \leq 0, \frac{\partial}{\partial t} F\left(x_{0}, t_{0}\right) \geq 0$, and $\nabla F\left(x_{0}, t_{0}\right)=0$. Hence the estimate

$$
\begin{aligned}
& \frac{2 a}{n} \frac{F^{2}}{t_{0}}-\frac{F}{t_{0}}-\frac{t_{0} n}{(1-a)} k^{2}-\frac{\alpha^{2}(0) n}{1-a} \frac{C^{2}}{t_{0}} \leq 0 \\
& \frac{2 a}{n} F^{2}-F-\frac{t_{0}^{2} n}{(1-a)} k^{2}-\frac{\alpha^{2}(0) n}{1-a} C^{2} \leq 0
\end{aligned}
$$


holds at $\left(x_{0}, t_{0}\right)$.

The quadratic formula implies that

$$
F\left(x_{0}, t_{0}\right) \leq \frac{n}{4 a}\left(1+\sqrt{1+\frac{8 a}{1-a} \cdot\left(t_{0}^{2} k^{2}+\alpha^{2}(0) C^{2}\right)}\right) .
$$

Choosing the following value for $a \in(0,1)$ (which happens to also minimize the RHS expression):

$$
a=\frac{1+\sqrt{2\left(t_{0}^{2} k^{2}+\alpha^{2}(0) C^{2}\right)}}{1+2 \sqrt{2\left(t_{0}^{2} k^{2}+\alpha^{2}(0) C^{2}\right)}}
$$

leads to the following inequality:

$$
\begin{aligned}
F\left(x_{0}, t_{0}\right) & \leq \frac{n}{4} \cdot\left(1+2 \sqrt{2\left(t_{0}^{2} k^{2}+\alpha^{2}(0) C^{2}\right)}\right) \\
& \leq \frac{n}{4} \cdot\left(1+4\left(t_{0} k+\alpha(0) C\right)\right)=n t_{0} k+n \alpha(0) C+\frac{n}{4}
\end{aligned}
$$

The fact that $\left(x_{0}, t_{0}\right)$ is a maximum point for $F$ on $M \times[0, \tau]$ enables us to conclude that

$$
F(x, \tau) \leq F\left(x_{0}, t_{0}\right) \leq n t_{0} k+n \alpha(0) C+\frac{n}{4} \leq \tau k n+n \alpha(0) C+\frac{n}{4}
$$

for all $x \in M$. Therefore, the estimate

$$
\frac{|\nabla u|^{2}}{u^{2}}-\frac{u_{t}}{u} \leq k n+\frac{C_{n}}{2 \tau}
$$

holds at $(x, \tau)\left(C_{n}=n / 2+4 n C \alpha(0)\right)$. Because the number $\tau \in(0, T]$ was chosen arbitrarily, this holds for all $t \in(0, T]$.

\subsection{Proof of Theorem 1.2.}

Proof. Let $f=\log u$ and $F=t\left(|\nabla f|^{2}-\beta f_{t}\right)$. By Lemma 2.1, one has that

$$
\begin{aligned}
\triangle F-F_{t} & \geq-2 \nabla f \cdot \nabla F-\left(|\nabla f|^{2}-\beta f_{t}\right)+\frac{2 a \beta t}{n}\left(|\nabla f|^{2}-f_{t}\right)^{2}-2 k_{1} \beta t|\nabla f|^{2} \\
& -\frac{\beta t n}{2 b} \max \left\{k_{1}^{2}, k_{2}^{2}\right\}-\frac{\beta \alpha^{2}(0) n}{2 b} \frac{C^{2}}{t}-2(\beta-1) \alpha(0) C|\nabla f|^{2}
\end{aligned}
$$

To make the computation easier to follow, let's assume $k_{2}=\max \left\{k_{1}, k_{2}\right\}$ and denote $\alpha(0)=\alpha$. Therefore the above inequality becomes:

$$
\begin{aligned}
\Delta F-F_{t} & \geq-2 \nabla f \cdot \nabla F-\left(|\nabla f|^{2}-\beta f_{t}\right)+\frac{2 a \beta t}{n}\left(|\nabla f|^{2}-f_{t}\right)^{2}-2 k_{1} \beta t|\nabla f|^{2} \\
& -\frac{\beta t n k_{2}}{2 b}-\frac{\beta \alpha^{2} n}{2 b} \frac{C^{2}}{t}-2(\beta-1) \alpha C|\nabla f|^{2}
\end{aligned}
$$

Following the classical technique, let $\tau \in(0, T], x_{0} \in M$ and fix $\bar{\Psi}(r, t)$ satisfying the conditions of Lemma 2.2. Further define $\Psi: M \times[0, T] \rightarrow \mathbb{R}$ by setting

$$
\Psi(x, t)=\bar{\Psi}\left(\operatorname{dist}\left(x, x_{0}, t\right), t\right) .
$$

We will the apply maximum principle to the expression $\left(\frac{\partial}{\partial t}-\Delta\right)(\Psi F)$ and we will establish (1.4) at $(x, \tau)$ for $x \in M$ such that $\operatorname{dist}\left(x, x_{0}, \tau\right)<\frac{\rho}{2}$. 
From the inequality (2.2) and some manipulations, one obtains:

$$
\begin{aligned}
\left(\Delta-\frac{\partial}{\partial t}\right)(\Psi F) \geq & -2 \nabla f \nabla(\Psi F)+2 F \nabla f \nabla \Psi \\
& +\left(\frac{2 a \beta t}{n}\left(|\nabla f|^{2}-f_{t}\right)^{2}-\left(|\nabla f|^{2}-\beta f_{t}\right)-2 k_{1} \beta t|\nabla f|^{2}\right) \Psi \\
& +\left(-\frac{\beta t n k_{2}}{2 b}-\frac{C^{2} \beta \alpha^{2} n}{2 b t}-2(\beta-1) \alpha C|\nabla f|^{2}\right) \Psi \\
& +(\Delta \Psi) F+2 \frac{\nabla \Psi}{\Psi} \nabla(\Psi F)-2 \frac{|\nabla \Psi|^{2}}{\Psi} F-\frac{\partial \Psi}{\partial t} F
\end{aligned}
$$

This holds in the region of $B_{\rho, T}$ where $\Psi(x, t)$ is smooth and strictly positive. Let $\left(x_{1}, t_{1}\right)$ be a maximum point for the function $\Psi F$ in the set $\left\{(x, t) \in M \times[0, \tau] \mid \operatorname{dist}\left(x, x_{0}, t\right) \leq \rho\right\}$. We may assume $(\Psi F)\left(x_{1}, t_{1}\right)>0$ without loss of generality. Note that if this is not the case, then $F(x, \tau) \leq 0$ and (1.4) is obvious at $(x, \tau)$ whenever $\operatorname{dist}\left(x, x_{0}, \tau\right)<\frac{\rho}{2}$. We may also assume that $\Psi(x, t)$ is smooth at $\left(x_{1}, t_{1}\right)$ due to a standard trick explained, for example, in [14, page 21 . Since $\left(x_{1}, t_{1}\right)$ is a maximum point, the equalities $\Delta(\Psi F)\left(x_{1}, t_{1}\right) \leq 0, \nabla(\Psi F)\left(x_{1}, t_{1}\right)=0$, and $(\Psi F)_{t}\left(x_{1}, t_{1}\right) \geq 0$ hold. Therefore (2.3) gives:

$$
\begin{aligned}
0 \geq & 2 F \nabla f \nabla \Psi+\left(\frac{2 a \beta t_{1}}{n}\left(|\nabla f|^{2}-f_{t}\right)^{2}-\left(|\nabla f|^{2}-\beta f_{t}\right)-2 k_{1} \beta t_{1}|\nabla f|^{2}\right) \Psi \\
+ & \left(-\frac{\beta t_{1} n k_{2}}{2 b}-\frac{C^{2} \beta \alpha^{2} n}{2 b t_{1}}-2(\beta-1) \alpha C|\nabla f|^{2}\right) \Psi \\
& +(\Delta \Psi) F-2 \frac{|\nabla \Psi|^{2}}{\Psi} F-\frac{\partial \Psi}{\partial t} F
\end{aligned}
$$

at $\left(x_{1}, t_{1}\right)$. We will use (2.4) to show that a certain quadratic expression in $\Psi F$ is nonpositive.

Recalling Lemma 2.2, together with the Laplacian comparison theorem, one can deduce that

$$
\begin{aligned}
-\frac{|\nabla \Psi|^{2}}{\Psi} & \geq-\frac{C_{\frac{1}{2}}^{2}}{\rho^{2}}, \\
\Delta \Psi & \geq-\frac{C_{\frac{1}{2}}}{\rho^{2}}-\frac{C_{\frac{1}{2}} \Psi^{\frac{1}{2}}}{\rho}(n-1) \sqrt{k_{1}} \operatorname{coth}\left(\sqrt{k_{1}} \rho\right) \geq-\frac{d_{1}}{\rho^{2}}-\frac{d_{1} \Psi^{\frac{1}{2}}}{\rho} \sqrt{k_{1}}
\end{aligned}
$$

at the point $\left(x_{1}, t_{1}\right)$ with $d_{1}$ a positive constant depending on $n$.

We will now find a bound for $\left(\Psi_{t}\right)\left(x_{1}, t_{1}\right)$. By definition of $\Psi$ :

$$
\begin{aligned}
\left(\Psi_{t}\right)\left(x_{1}, t_{1}\right)= & \frac{\partial \bar{\Psi}}{\partial t}\left(\operatorname{dist}\left(x_{1}, x_{0}, t_{1}\right), t_{1}\right) \\
& +\frac{\partial \bar{\Psi}}{\partial r}\left(\operatorname{dist}\left(x_{1}, x_{0}, t_{1}\right), t_{1}\right)\left(\frac{\partial}{\partial t} \operatorname{dist}\left(x_{1}, x_{0}, t_{1}\right)\right) .
\end{aligned}
$$

Using the fact that the function $\bar{\Psi}(r, t)$ satisfies the conditions listed in Lemma 2.2, the inequality

$$
\left|\frac{\partial \bar{\Psi}}{\partial r}\left(\operatorname{dist}\left(x_{1}, x_{0}, t_{1}\right), t_{1}\right)\right| \leq \frac{C_{\frac{1}{2}}}{\rho} \Psi^{\frac{1}{2}}\left(x_{1}, t_{1}\right)
$$


holds with $C_{\frac{1}{2}}>0$. For the estimate of the derivative of the distance, notice that:

$$
\left|\frac{\partial}{\partial t} \operatorname{dist}\left(x_{1}, x_{0}, t_{1}\right)\right| \leq \sup \int_{0}^{\operatorname{dist}\left(x_{1}, x_{0}, t_{1}\right)}\left|\operatorname{Ric}\left(\frac{d}{d s} \zeta(s), \frac{d}{d s} \zeta(s)\right)\right| d s \leq k_{2} \operatorname{dist}\left(x_{1}, x_{0}, t_{1}\right) \leq k_{2} \rho .
$$

Here Ric represents the Ricci curvature of $g\left(x, t_{1}\right)$ and the supremum is taken over all the minimal geodesics $\zeta(s)$, with respect to $g\left(x, t_{1}\right)$, that connect $x_{0}$ to $x_{1}$ and are parametrized by arclength; see, e.g., [16, Proof of Lemma 8.28].

Moreover, again by Lemma 2.2

$$
\left|\frac{\partial \bar{\Psi}}{\partial t}\left(\operatorname{dist}\left(x_{1}, x_{0}, t_{1}\right), t_{1}\right)\right| \leq \frac{c_{2} \bar{\Psi}^{1 / 2}}{\tau} \leq \frac{c_{2} \Psi^{1 / 2}}{\tau}
$$

for a positive constant $c_{2}$.

Therefore, we can conclude that there exists $\bar{C}>0$ such that the inequality

$$
-\frac{\partial \Psi}{\partial t} \geq-\frac{\bar{C} \Psi^{\frac{1}{2}}}{\tau}-C_{\frac{1}{2}} k_{2} \Psi^{\frac{1}{2}}
$$

holds true. Combining this with (2.4), we find the estimate

$$
\begin{aligned}
0 \geq & -2 F|\nabla f||\nabla \Psi|+\left(\frac{2 a \beta t_{1}}{n}\left(|\nabla f|^{2}-f_{t}\right)^{2}-\left(|\nabla f|^{2}-\beta f_{t}\right)-2 k_{1} \beta t_{1}|\nabla f|^{2}\right) \Psi \\
+ & \left(-\frac{\beta t_{1} n k_{2}}{2 b}-\frac{C^{2} \beta \alpha^{2} n}{2 b t_{1}}-2(\beta-1) \alpha C|\nabla f|^{2}\right) \Psi \\
& +d_{2}\left(-\frac{1}{\rho^{2}}-\frac{\Psi^{\frac{1}{2}}}{\rho} \sqrt{k_{1}}-\frac{\Psi^{\frac{1}{2}}}{\tau}-k_{2} \Psi^{\frac{1}{2}}\right) F
\end{aligned}
$$

at $\left(x_{1}, t_{1}\right)$. Here, $d_{2}$ is equal to $\max \left\{3 d_{1}, C_{\frac{1}{2}}, 3 C_{\frac{1}{2}}^{2}, \bar{C}\right\}$. Let us now multiply by $t_{1} \Psi$, rearrange the terms and obtain:

$$
\begin{aligned}
0 \geq & -2 t_{1} F \frac{C_{\frac{1}{2}} \Psi^{\frac{3}{2}}}{\rho}|\nabla f|+\frac{2 t_{1}^{2}}{n}\left(a \beta\left(\Psi|\nabla f|^{2}-\Psi f_{t}\right)^{2}-n k_{1} \beta \Psi^{2}|\nabla f|^{2}-\frac{n^{2} \beta}{4 b} k_{2}^{2} \Psi^{2}\right) \\
+ & \left(-\frac{C^{2} \beta \alpha^{2} n^{2}}{4 b t_{1}^{2}} \Psi^{2}-\frac{(\beta-1) \alpha C|\nabla f|^{2}}{t_{1}} \Psi^{2}\right) \\
& +d_{2} t_{1}\left(-\frac{1}{\rho^{2}}-\frac{\sqrt{k_{1}}}{\rho}-\frac{1}{\tau}-k_{2}\right)(\Psi F)-\Psi F
\end{aligned}
$$

at $\left(x_{1}, t_{1}\right)$. Our next step is to estimate the first two terms in the right-hand side. The procedure is standard (see [13]). 
Define $y=\Psi|\nabla f|^{2}$ and $z=\Psi f_{t}$. It follows that $y^{\frac{1}{2}}(y-\beta z)=\frac{\Psi^{\frac{3}{2}} F|\nabla f|}{t}$ when $t \neq 0$, which yields

$$
\begin{array}{r}
-2 t_{1} F \frac{C_{\frac{1}{2}} \Psi^{\frac{3}{2}}}{\rho}|\nabla f|+\frac{2 t_{1}^{2}}{n}\left(a \beta\left(\Psi|\nabla f|^{2}-\Psi f_{t}\right)^{2}-n k_{1} \beta \Psi^{2}|\nabla f|^{2}-\frac{n^{2} \beta}{4 b} k_{2}^{2} \Psi^{2}\right) \\
+\left(-\frac{C^{2} \beta \alpha^{2} n^{2}}{4 b t_{1}^{2}} \Psi^{2}-\frac{(\beta-1) \alpha C|\nabla f|^{2}}{t_{1}} \Psi^{2}\right) \\
=\frac{2 t_{1}^{2}}{n}\left(-\frac{n C_{\frac{1}{2}}}{\rho} y^{\frac{1}{2}}(y-\beta z)+a \beta(y-z)^{2}-n k_{1} \beta y-\frac{n^{2} \beta}{4 b} k_{2}^{2} \Psi^{2}-\frac{C^{2} \beta \alpha^{2} n^{2}}{4 b t_{1}^{2}} \Psi^{2}-\frac{(\beta-1) \alpha C}{t_{1}} y \Psi\right) .
\end{array}
$$

Observe that

$$
(y-z)^{2}=\frac{1}{\beta^{2}}(y-\beta z)^{2}+\frac{(\beta-1)^{2}}{\beta^{2}} y^{2}+\frac{2(\beta-1)}{\beta^{2}} y(y-\beta z)
$$

and plug this into the above:

$$
\begin{array}{r}
\frac{2 t_{1}^{2}}{n}\left(-\frac{n C_{\frac{1}{2}}}{\rho} y^{\frac{1}{2}}(y-\beta z)+a \beta(y-z)^{2}-n k_{1} \beta y-\frac{n^{2} \beta}{4 b} k_{2}^{2} \Psi^{2}-\frac{C^{2} \beta \alpha^{2} n^{2}}{4 b t_{1}^{2}} \Psi^{2}-\frac{(\beta-1) \alpha C}{t_{1}} y \Psi\right) \\
=\frac{2 t_{1}^{2}}{n}\left(-\frac{n C_{\frac{1}{2}}}{\rho} y^{\frac{1}{2}}(y-\beta z)+\frac{a(y-\beta z)^{2}}{\beta}+\frac{a}{\beta}(\beta-1)^{2} y^{2}+\frac{2(\beta-1) a}{\beta} y(y-\beta z)\right) \\
+\frac{2 t_{1}^{2}}{n}\left(-n k_{1} \beta y-\frac{n^{2} \beta}{4 b} k_{2}^{2} \Psi^{2}-\frac{C^{2} \beta \alpha^{2} n^{2}}{4 b t_{1}^{2}} \Psi^{2}-\frac{(\beta-1) \alpha C}{t_{1}} y \Psi\right)
\end{array}
$$

Using the inequality $\kappa_{1} v^{2}-\kappa_{2} v \geq-\frac{\kappa_{2}^{2}}{4 \kappa_{1}}$, which holds for $\kappa_{1}, \kappa_{2}>0$ and $v \in \mathbb{R}$, we obtain:

$$
\begin{array}{r}
\frac{2(\beta-1) a}{\beta} y(y-\beta z)-\frac{n C_{\frac{1}{2}}}{\rho} y^{\frac{1}{2}}(y-\beta z) \geq-\frac{n^{2} C_{\frac{1}{2}}^{2} \beta(y-\beta z)}{\rho^{2} 8 a(\beta-1)} \\
\frac{a}{\beta}(\beta-1)^{2} y^{2}-\left(n k_{1} \beta+\frac{(\beta-1) \alpha C}{t_{1}} \Psi\right) y \geq-\frac{\beta\left(n k_{1} \beta+\frac{(\beta-1) \alpha C}{t_{1}} \Psi\right)^{2}}{4 a(\beta-1)^{2}}
\end{array}
$$

It then follows that:

$$
\begin{array}{r}
-2 t_{1} F \frac{C_{\frac{1}{2}} \Psi^{\frac{3}{2}}}{\rho}|\nabla f|+\frac{2 t_{1}^{2}}{n}\left(a \beta\left(\Psi|\nabla f|^{2}-\Psi f_{t}\right)^{2}-n k_{1} \beta \Psi^{2}|\nabla f|^{2}-\frac{n^{2} \beta}{4 b} k_{2}^{2} \Psi^{2}\right) \\
+\left(-\frac{C^{2} \beta \alpha^{2} n^{2}}{4 b t_{1}^{2}} \Psi^{2}-\frac{(\beta-1) \alpha C|\nabla f|^{2}}{t_{1}} \Psi^{2}\right) \\
\geq \frac{2 t_{1}^{2}}{n}\left(\frac{a(y-\beta z)^{2}}{\beta}-\frac{n^{2} \beta}{4 b} k_{2}^{2} \Psi^{2}-\frac{C^{2} \beta \alpha^{2} n^{2}}{4 b t_{1}^{2}} \Psi^{2}-\frac{n^{2} C_{\frac{1}{2}}^{2} \beta(y-\beta z)}{\rho^{2} 8 a(\beta-1)}-\frac{\beta\left(n k_{1} \beta+\frac{(\beta-1) \alpha C}{t_{1}} \Psi\right)^{2}}{4 a(\beta-1)^{2}}\right)
\end{array}
$$


Recall that $t(y-\beta z)=\Psi F$ so inequality (2.8) becomes:

$$
\begin{aligned}
0 \geq & \frac{2 a}{n \beta}(\Psi F)^{2}+\left[-\frac{n d_{3} t_{1}}{\rho^{2}}\left(\frac{\beta}{a(\beta-1)}+1+\rho \sqrt{k_{1}}+\frac{\rho^{2}}{\tau}+\rho^{2} k_{2}\right)-1\right](\Psi F) \\
& -\frac{\beta\left(n k_{1} \beta+\frac{(\beta-1) \alpha C}{t_{1}} \Psi\right)^{2}}{2 a n(\beta-1)^{2}} t_{1}^{2}-\frac{\beta n}{2 b} t_{1}^{2} k_{2}^{2} \Psi^{2}-\frac{C^{2} \beta \alpha^{2} n}{2 b} \Psi^{2} \\
\geq & \frac{2 a}{n \beta}(\Psi F)^{2}+\left(-\frac{d_{4} t_{1}}{\rho^{2}}\left(\frac{\beta}{a(\beta-1)}+\frac{\rho^{2}}{\tau}+\rho^{2} \bar{k}\right)-1\right)(\Psi F) \\
& -\frac{\beta\left(n k_{1} \beta+\frac{(\beta-1) \alpha C}{t_{1}} \Psi\right)^{2}}{2 a n(\beta-1)^{2}} t_{1}^{2}-\left(\frac{\beta n}{2 b} t_{1}^{2} k_{2}^{2}+\frac{C^{2} \beta \alpha^{2} n}{2 b}\right) \Psi^{2}
\end{aligned}
$$

at $\left(x_{1}, t_{1}\right)$ with $d_{3}=\max \left\{4 d_{2}, C_{1 / 2}\right\}, d_{4}=n d_{3}$ and $\bar{k}=\max \left\{k_{1}, k_{2}\right\}$. The expression in the last two lines is a polynomial in $\Psi F$ of degree 2 . The quadratic formula yields:

$$
\begin{aligned}
\Psi F & \leq \frac{n \beta}{4 a}\left[2 \frac{d_{4} t_{1}}{\rho^{2}}\left(\frac{\beta}{a(\beta-1)}+\frac{\rho^{2}}{\tau}+\rho^{2} \bar{k}\right)+2\right] \\
& +\frac{n \beta}{4 a}\left[\frac{2}{n(\beta-1)}\left(n k_{1} b+\frac{(\beta-1) \alpha C}{t_{1}} \Psi\right) t_{1}+2 \sqrt{\frac{a}{b}}\left(t_{1} k_{2}+C \alpha\right) \Psi\right] \\
& =\frac{n \beta}{2 a}\left[\frac{d_{4} t_{1}}{\rho^{2}}\left(\frac{\beta}{a(\beta-1)}+\frac{\rho^{2}}{\tau}+\rho^{2} \bar{k}\right)+1+\frac{k_{1} b t_{1}}{(\beta-1)}+\left(\frac{\alpha C}{n}+\sqrt{\frac{a}{b}}\left(t_{1} k_{2}+C \alpha\right)\right) \Psi\right]
\end{aligned}
$$

at $\left(x_{1}, t_{1}\right)$. We will now use this estimate to obtain a bound on $F(x, \tau)$ for an appropriate range of $x \in M$.

By definition $\Psi(x, \tau)=1$ whenever $\operatorname{dist}\left(x, x_{0}, \tau\right)<\frac{\rho}{2}$. Moreover, $\left(x_{1}, t_{1}\right)$ is a maximum point for $\Psi F$ in the set $\left\{(x, t) \in M \times[0, \tau] \mid \operatorname{dist}\left(x, x_{0}, t\right) \leq \rho\right\}$. Hence

$$
\begin{aligned}
F(x, \tau) & =(\Psi F)(x, \tau) \leq(\Psi F)\left(x_{1}, t_{1}\right) \\
& \leq \frac{n \beta}{2 a} \cdot \frac{d_{4} \tau}{\rho^{2}}\left(\frac{\beta}{a(\beta-1)}+\frac{\rho^{2}}{\tau}+\rho^{2} \bar{k}\right)+\frac{n \beta}{2 a}\left(1+\frac{\alpha C}{n}+\sqrt{\frac{a}{b}} \alpha C\right)+\frac{n \beta}{2 a} \cdot \frac{k_{1} b \tau}{(\beta-1)}+\frac{n \beta k_{2}}{2 \sqrt{a b}} \tau
\end{aligned}
$$

for all $x \in M$ such that $\operatorname{dist}\left(x, x_{0}, \tau\right)<\frac{\rho}{2}$. Since $\tau \in(0, T]$ was chosen arbitrarily, this formula implies

$$
\begin{aligned}
\left(|\nabla f|^{2}-\beta f_{t}\right)(x, t) & \leq \frac{n \beta}{2 a} \cdot \frac{d_{4}}{\rho^{2}}\left(\frac{\beta}{a(\beta-1)}+\frac{\rho^{2}}{t}+\rho^{2} \bar{k}\right) \\
& +\frac{n \beta}{2 a t}\left(1+\frac{\alpha C}{n}+\sqrt{\frac{a}{b}} \alpha C\right)+\frac{n \beta}{2 a} \cdot \frac{k_{1} b}{(\beta-1)}+\frac{n \beta k_{2}}{2 \sqrt{a b}} \\
& =\frac{n \beta}{2 a} \cdot \frac{d_{4}}{\rho^{2}} \cdot \frac{\beta}{a(\beta-1)}+\frac{n \beta}{2 a t}\left(d_{4}+1+\frac{\alpha C}{n}+\sqrt{\frac{a}{b}} \alpha C\right) \\
& +\frac{n \beta}{2 a} \cdot \frac{k_{1} b}{(\beta-1)}+\frac{n \beta}{2}\left(\frac{d_{4} \bar{k}}{a}+\frac{k_{2}}{\sqrt{a b}}\right)
\end{aligned}
$$

for $(x, t) \in B_{\frac{\rho}{2}, T}$, as long as $t \neq 0$.

If we set $a=\frac{1}{2 \beta}$ and $b=\frac{1}{4 \beta}$, then the above becomes: 


$$
\begin{aligned}
\left(|\nabla f|^{2}-\beta f_{t}\right)(x, t) & \leq n \beta^{2} \cdot \frac{d_{4}}{\rho^{2}} \cdot \frac{2 \beta^{2}}{(\beta-1)}+\frac{n \beta^{2}}{t}\left(d_{4}+1+\frac{\alpha C}{n}+\sqrt{2} \alpha C\right) \\
& +\frac{n \beta k_{1}}{4(\beta-1)}+n \beta^{2}\left(d_{4} \bar{k}+2 k_{2}\right)
\end{aligned}
$$

Define the constant $C^{\prime}$ as $\max \left\{2 n d_{4}, n\left(d_{4}+1+\frac{\alpha C}{n}+\sqrt{2} \alpha C\right), n\left(d_{4}+2\right)\right\}$, estimate (1.4) will follow:

$$
\left(|\nabla f|^{2}-\beta f_{t}\right)(x, t) \leq C^{\prime} \beta^{2} \cdot\left(\frac{\beta^{2}}{\rho(\beta-1)}+\frac{1}{t}+\max \left\{k_{1}, k_{2}\right\}\right)+\frac{n \beta k_{1}}{4(\beta-1)}
$$

for $(x, t) \in B_{\frac{\rho}{2}, T}$, as long as $t \neq 0$.

\section{HARNACK INEQUALITIES}

In this section, we will prove two Harnack inequalities as a direct application to the gradient estimates obtained in section 2, as for example, [14, Chapter IV]. One can find similar Harnack inequalities for the heat equation under the Ricci flow in the papers [8, 7, 10].

Given $x_{1}, x_{2} \in M$ and $t_{1}, t_{2} \in(0, T)$ satisfying $t_{1}<t_{2}$, define

$$
\Gamma\left(x_{1}, t_{1}, x_{2}, t_{2}\right)=\inf \int_{t_{1}}^{t_{2}}\left|\frac{d}{d t} \gamma(t)\right|^{2} d t
$$

where the infimum is taken over the set $\Theta\left(x_{1}, t_{1}, x_{2}, t_{2}\right)$ of all the smooth paths $\gamma:\left[t_{1}, t_{2}\right] \rightarrow M$ that connect $x_{1}$ to $x_{2}$ and the norm $|\cdot|$ is calculated at time $t$.

The way we will prove the Harnack inequalities is to use the above gradient estimates together with the next lemma:

Lemma 3.1. Suppose $(\phi(x, t), g(x, t))_{t \in[0, T]}$ is a solution to the $(R H)_{\alpha}$ flow (1.1) such that $M$ is a complete manifold. Let $u: M \times[0, T] \rightarrow \mathbb{R}$ be a smooth positive function satisfying the heat equation (1.2). Define $f=\log u$ and assume that

$$
\frac{\partial f}{\partial t} \geq \frac{1}{A_{1}}\left(|\nabla f|^{2}-A_{2}-\frac{A_{3}}{t}\right), \quad x \in M, t \in(0, T],
$$

for some $A_{1}, A_{2}, A_{3}>0$. Then the inequality

$$
u\left(x_{2}, t_{2}\right) \geq u\left(x_{1}, t_{1}\right)\left(\frac{t_{2}}{t_{1}}\right)^{-\frac{A_{3}}{A_{1}}} \exp \left(-\frac{A_{1}}{4} \Gamma\left(x_{1}, t_{1}, x_{2}, t_{2}\right)-\frac{A_{2}}{A_{1}}\left(t_{2}-t_{1}\right)\right)
$$

holds for all $\left(x_{1}, t_{1}\right),\left(x_{2}, t_{2}\right) \in M \times(0, T)$ such that $t_{1}<t_{2}$.

Proof. The technique used to prove this result is traditional; see, for example, [14, Chapter IV] and [10, 9]. Let $\gamma(t) \in \Theta\left(x_{1}, t_{1}, x_{2}, t_{2}\right)$ be a path connecting $\left(x_{1}, t_{1}\right)$ and $\left(x_{2}, t_{2}\right)$. 
Taking the time derivative at the point $(\gamma(t), t)$, one obtains:

$$
\begin{aligned}
\frac{d}{d t} f(\gamma(t), t) & =\nabla f(\gamma(t), t) \frac{d}{d t} \gamma(t)+\left.\frac{\partial}{\partial s} f(\gamma(t), s)\right|_{s=t} \\
& \geq-|\nabla f(\gamma(t), t)|\left|\frac{d}{d t} \gamma(t)\right|+\frac{1}{A_{1}}\left(|\nabla f(\gamma(t), t)|^{2}-A_{2}-\frac{A_{3}}{t}\right) \\
& \geq-\frac{A_{1}}{4}\left|\frac{d}{d t} \gamma(t)\right|^{2}-\frac{1}{A_{1}}\left(A_{2}+\frac{A_{3}}{t}\right), \quad t \in\left[t_{1}, t_{2}\right] .
\end{aligned}
$$

where for the last step one applies the inequality $\kappa_{1} v^{2}-\kappa_{2} v \geq-\frac{\kappa_{2}^{2}}{4 \kappa_{1}}\left(\right.$ for $\kappa_{1}, \kappa_{2}>0$ and $v \in \mathbb{R})$.

Next, let us integrate from $t_{1}$ to $t_{2}$

$$
\begin{aligned}
f\left(x_{2}, t_{2}\right)-f\left(x_{1}, t_{1}\right) & =\int_{t_{1}}^{t_{2}} \frac{d}{d t} f(\gamma(t), t) d t \\
& \geq-\frac{A_{1}}{4} \int_{t_{1}}^{t_{2}}\left|\frac{d}{d t} \gamma(t)\right|^{2} d t-\frac{A_{2}}{A_{1}}\left(t_{2}-t_{1}\right)-\frac{A_{3}}{A_{1}} \ln \frac{t_{2}}{t_{1}} .
\end{aligned}
$$

Now the conclusion follows by exponentiating.

We will obtain two Harnack inequalities for (1.1)-(1.2), one for noncompact manifolds, one for compact.

Theorem 3.2. Let $(g(x, t), \phi(x, t))_{t \in[0, T]}$ be a solution to the $(R H)_{\alpha}$ flow (1.1), where $M^{n}$ is complete and $\alpha(t)$ is a non-increasing function in time, bounded from below by $\bar{\alpha}$. Suppose $-k_{1} g(x, t) \leq \operatorname{Ric}(x, t) \leq k_{2} g(x, t)$ for some $k_{1}, k_{2}>0$ and that $0 \leq \nabla_{i} \phi \nabla_{j} \phi \leq \frac{C}{t} g_{i j}$, for all $i, j \in\{1,2, \ldots, n\}$ ( $C$ being a constant depending on $n$ and $\bar{\alpha})$. Consider a smooth positive function $u: M \times[0, T] \rightarrow \mathbb{R}$ solving the heat equation $\triangle u=u_{t}$. Given $\beta>1$, the estimate

$u\left(x_{2}, t_{2}\right) \geq u\left(x_{1}, t_{1}\right)\left(\frac{t_{2}}{t_{1}}\right)^{-C^{\prime} \beta} \exp \left(-\frac{\beta}{4} \Gamma\left(x_{1}, t_{1}, x_{2}, t_{2}\right)-\left(C^{\prime} \beta \max \left\{k_{1}, k_{2}\right\}+\frac{n \beta k_{1}}{4(\beta-1)}\right)\left(t_{2}-t_{1}\right)\right)$

holds for all $\left(x_{1}, t_{1}\right),\left(x_{2}, t_{2}\right) \in M \times(0, T)$ such that $t_{1}<t_{2}$. The constant $C^{\prime}$ comes from Theorem 1.2.

Proof. Let $\rho$ go to infinity in (1.4) and obtain that

$$
\frac{u_{t}}{u} \geq \frac{1}{\beta}\left(\frac{|\nabla u|^{2}}{u^{2}}-\frac{C^{\prime} \beta^{2}}{t}-\left(C^{\prime} \beta^{2} \max \left\{k_{1}, k_{2}\right\}+\frac{n \beta^{2} k_{1}}{4(\beta-1)}\right)\right)
$$

on $M \times(0, T]$. To get the conclusion, apply Lemma 3.1,

Theorem 3.3. Let $(g(x, t), \phi(x, t))_{t \in[0, T]}$ is a solution to the $(R H)_{\alpha}$ flow (1.1), where the manifold $M^{n}$ is compact and $\alpha(t)$ is a non-increasing function in time, bounded from below by $\bar{\alpha}$. Assume that $0 \leq \operatorname{Ric}(x, t) \leq k g(x, t)$ for some $k>0$ and all $(x, t) \in M \times[0, T]$. Moreover suppose that $0 \leq \nabla_{i} \phi \nabla_{j} \phi \leq \frac{C}{t} g_{i j}$, for all $i, j \in\{1,2, \ldots, n\}$ (C being a constant depending on $n$ and $\bar{\alpha})$. Let $u$ be a smooth positive function $u: M \times[0, T] \rightarrow \mathbb{R}$ satisfying 
the heat equation $u_{t}=\triangle u$. The estimate

$$
u\left(x_{2}, t_{2}\right) \geq u\left(x_{1}, t_{1}\right)\left(\frac{t_{2}}{t_{1}}\right)^{-\frac{C_{n}}{2}} \exp \left(-\frac{1}{4} \Gamma\left(x_{1}, t_{1}, x_{2}, t_{2}\right)-k n\left(t_{2}-t_{1}\right)\right)
$$

holds for all $\left(x_{1}, t_{1}\right) \in M \times(0, T)$ and $\left(x_{2}, t_{2}\right) \in M \times(0, T)$ as long as $t_{1}<t_{2}$. The constant $C_{n}$ is the same as in Theorem 1.1.

Proof. Theorem 1.1 implies

$$
\frac{u_{t}}{u} \geq \frac{|\nabla u|^{2}}{u^{2}}-k n-\frac{C_{n}}{2 t}, \quad x \in M, t \in(0, T] .
$$

Use again Lemma 3.1 to finish the proof.

3.1. Concluding remarks. We have obtained Li-Yau gradient estimates (and as an application Harnack inequalities) for the solution of the heat equation under the Ricci-harmonic flow, under a slightly stronger assumption on the covariant derivatives of the harmonic map. The results show a similar behavior of the heat function as in the classical (non-evolving) case. In the future, one may want to study the behavior in an even more general setting, for example only a lower bound on the Ricci curvature and no condition on the covariant derivatives of the harmonic map.

\section{REFERENCES}

[1] R. Mueller. Ricci flow coupled with harmonic map flow. Ann. Sci. Ec. Norm. Sup., 4(45):101-142, 2012.

[2] B. List. Evolution of an extended ricci flow system. Comm. Anal. Geom., 16(5):1007-1048, 2008.

[3] M.B. Williams. Stability of solutions of certain extended ricci flow systems. ArXiv:, (1301).

[4] H. Tran. Harnack estimates for ricci flow on a warped product. ArXiv:, (1211).

[5] R.S. Hamilton. The formation of singularities in the Ricci Flow. Surv. Differ. Geom., vol. II, International Press, Cambridge, MA, 1995.

[6] Q. Zhang. Some gradient estimates for the heat equation on domains and for an equation by perelman. Int. Math. Res. Not., 2006:1-39, 2006.

[7] L. Ni. Ricci flow and nonnegativity of sectional curvature. Math. Res. Lett., 11:883-904, 2004.

[8] C.M. Guenther. The fundamental solution on manifolds with time-dependent metrics. J. Geom. Anal., 12:425-436, 2002.

[9] X. Cao and R. Hamilton. Differential harnack estimates for time-dependent heat equations with potentials. Geom. Funct. Anal., 2009.

[10] M.Bailesteanu, X. Cao, and A. Pulemotov. Gradient estimates for the heat equation under the ricci flow. J. Funct. Anal., 2009.

[11] S. Liu. Gradient estimates for solutions of the heat equation under ricci flow. Pacific J. Math., 243(1):165-180, 2009.

[12] J. Sun. Gradient estimates for positive solutions of the heat equation under geometric flow. Pacific J. Math., 253(1):489-510, 2011.

[13] P. Li and S.-T. Yau. On the parabolic kernel of the schroedinger operator. Acta Math., 156:153-201, 1986.

[14] R. Schoen and S.-T. Yau. Lectures on differential geometry. International Press, Cambridge, MA, 1994.

[15] P. Souplet and Q. Zhang. Sharp gradient estimate and yau's liouville theorem for the heat equation on noncompact manifolds. Bull. London Math. Soc., 38:1045-1053, 2006.

[16] B. Chow, P. Lu, and L. Ni. Hamilton's Ricci flow. American Mathematical Society, Providence, RI; Science Press, New York, 2006. 